\title{
An Ultra-thin Multiband Terahertz Metamaterial Absorber and Sensing Applications
}

Jinjun Bai ( $\nabla$ baijinjun@tiangong.edu.cn )

Tiangong University https://orcid.org/0000-0002-3395-6258

\section{Wei Shen}

Tiangong University

\section{Shasha Wang}

Tiangong University

\section{Meilan Ge}

Tiangong University

\section{Tingting Chen}

Tiangong University

\section{Pengyan Shen}

Tiangong University

\section{Shengjiang Chang}

Nankai University

\section{Research Article}

Keywords: Multiband, Metamaterial absorber, Terahertz, Sensing

Posted Date: May 11th, 2021

DOI: https://doi.org/10.21203/rs.3.rs-475213/v1

License: (c) (i) This work is licensed under a Creative Commons Attribution 4.0 International License.

Read Full License

Version of Record: A version of this preprint was published at Optical and Quantum Electronics on August 14th, 2021. See the published version at https://doi.org/10.1007/s11082-021-03180-8. 


\title{
An ultra-thin multiband terahertz metamaterial absorber and sensing applications
}

Jinjun Bai ${ }^{1}$, Wei Shen ${ }^{1}$, Shasha Wang ${ }^{1}$, Meilan $\mathrm{Ge}^{1}$, Tingting Chen ${ }^{1}$, Pengyan Shen ${ }^{1}$, Shengjiang Chang ${ }^{2}$

${ }^{1}$ Tianjin Key Laboratory of Optoelectronic Detection Technology and Systems, International Research Center for Photonics, School of electrical and electronic Engineering, Tiangong University, Tianjin 300387, China.

${ }^{2}$ Institute of Modern Optics, Nankai University, Tianjin 300071, China.

E-mail: *wangshasha@tiangong.edu.cn, *baijinjun@ tiangong.edu.cn

\begin{abstract}
We propose an ultra-thin multiband terahertz metamaterial absorber, whose thickness is only $3.8 \mu \mathrm{m}$. Simulation results show that we can get four narrow absorption peaks with near-perfect absorption in the $4.5 \mathrm{THz}-6.0 \mathrm{THz}$ frequency range. The resonance absorption mechanism is interpreted by the electromagnetic field energy distributions at resonance frequency. Moreover, we also analyze the sensing performances of the absorber in the refractive index and the thickness of the analyte. The refractive index and thickness sensitivities of the sensor are 0.471THz/RIU, 36.594THz/RIU and the FOMs are $8.887 \mathrm{RIU}^{-1}$, 938.308RIU ${ }^{-1}$, respectively. The absorber has potential applications in photodetector, multi-spectral imaging and biosensors.
\end{abstract}

Keywords: Multiband, Metamaterial absorber, Terahertz, Sensing 


\section{Introduction}

In recent years, with the development of terahertz $(\mathrm{THz})$ radiation, detection technology, and the proposed functional devices, $\mathrm{THz}$ technology has presented promising applications in imaging [1], communication [2], biomedicine [3], etc. However, the lack of natural strong absorption material has limited the development of terahertz detector. Researchers found that metamaterials can strongly absorb incident radiations at the resonant frequency [4]. Thus, the exploration of metamaterial-based absorbers has been becoming a hot topic in $\mathrm{THz}$ field.

The first $\mathrm{THz}$ metamaterial absorber, having an experimental absorptivity of $70 \%$ at $1.3 \mathrm{THz}$, was demonstrated by H.Tao et al [5]. Since then, researchers investigated various metamaterial absorbers at different bandwidth including narrowband [6-8], multiband [9-12], broadband [13-15] and ultra-broadband [16-18]. The absorption bandwidth is one of the most important factors in the applications of the devices. Broadband absorbers are mainly used to solar cells [19] and photodetectors [20, 21]. Narrowband absorbers are mostly applied in sensing [22] and thermal emitter [23]. And absorbers with multiple absorption peaks are required in some applications, such as multispectral imaging [24, 25] and sensing [26-28]. The resonant structure with multiband absorption may be formed by single resonant cell [9, 11, 29], transverse nested multiple resonant cells $[12,30,31]$ and longitudinal stacked multiple resonant cells [32-34]. However, these absorbers have the disadvantages of being complicated, too thick, and difficult to integrate.

In this paper, we propose an ultra-thin multiband terahertz metamaterial absorber of only $3.8 \mu \mathrm{m}$ thickness. The absorber consists of a gold substrate layer and a subwavelength silicon disk. Using finite-difference time-domain (FDTD) method, the designed absorber has four perfect absorption peaks in the $4.5 \mathrm{THz}-6.0 \mathrm{THz}$ frequency range. In addition, we also analyze the performances of the absorber in refractive index and thickness sensing. The refractive index and thickness sensitivities of the sensor are $0.471 \mathrm{THz} / \mathrm{RIU}, 36.594 \mathrm{THz} / \mathrm{RIU}$ and the FOMs are $8.887 \mathrm{RIU}^{-1}$, 938.308RIU ${ }^{-1}$ respectively.

\section{Results and discussion}

Fig. 1(a) shows the periodic structure of the absorber, it is composed of a gold substrate layer, a dielectric layer (Polyimide), a silicon disk. The period of the 
structure is $P=80 \mu \mathrm{m}$. The gold has the thickness of $t_{1}=0.2 \mu \mathrm{m}$, and its conductivity is $4.09 \times 10^{7} \mathrm{~S} / \mathrm{m}$. The thickness and dielectric constant of polyimide are set as $t_{2}$, and $3.9+i 0.09$. The silicon disk has the radius of $r$, the thickness of $t_{3}$, and the dielectric constant of 11.7. Our results are obtained through the FDTD simulations, where the period structures are illuminated by a normally incident plane wave with the electric field parallel to the $x$-axis. The unit cell boundary condition is set in the $x$-direction and $y$-direction. The formula of absorption is as follows [35]: $A(f)=1-R(f)-T(f)$, where $R(f)=\left|S_{11}\right|^{2}$ and $T(f)=\left|S_{21}\right|^{2} \quad$ are reflectivity and transmissivity, respectively. And $S_{11}$ and $S_{21}$ are reflection coefficient and transmission coefficient, respectively. The thickness of the gold substrate layer $(0.2 \mu \mathrm{m})$ is larger than skin depth of incident electromagnetic wave, so the transmissivity is close to zero. The $A(f)$ achieves prefect absorption, which only needs to satisfy the reflectivity $R(f)=\left|S_{11}\right|^{2}=0$.

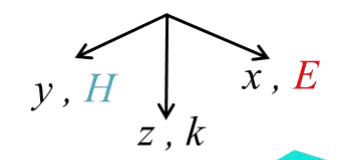

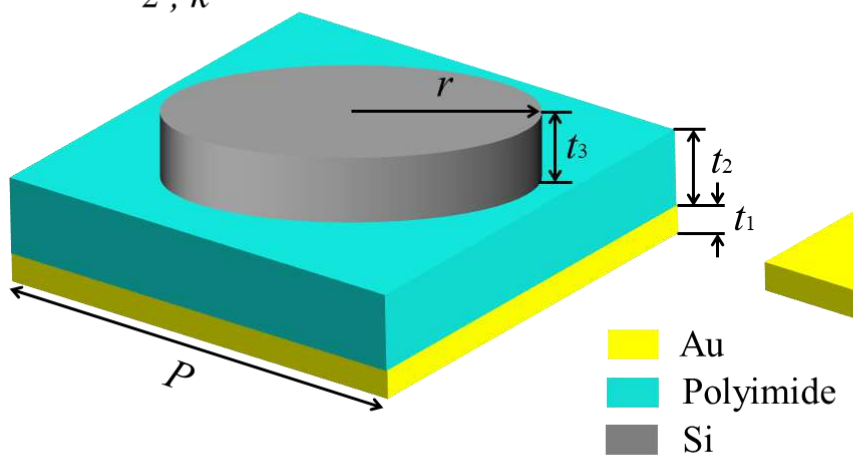

(a)

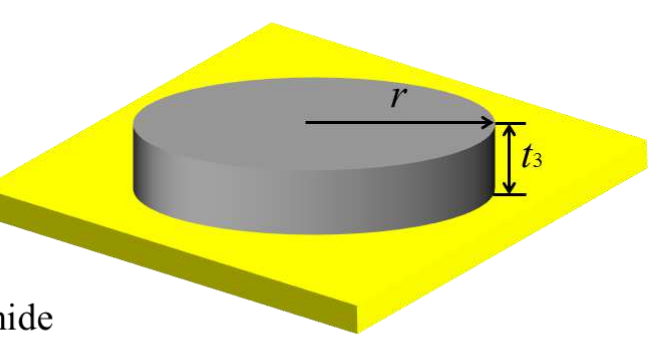

(b)

Fig. 1 (a) The periodic structure schematic diagram of a tri-layer terahertz absorber. The top-down for a silicon disk (gray), polyimide (blue) and gold (yellow). (b) The periodic structure schematic diagram of a bi-layer terahertz absorber.

According to the theory of transmission line, the reflection and transmission coefficients depends on the input impedance $Z(f)=\sqrt{\mu(f)+\varepsilon(f)}=z_{1}+i z_{2}$. When the input impedance matches the free space impedance, i.e. $z_{1}=1, z_{2}=0$, the 
reflection coefficient equals zero. The normalized input impedance $(\mathrm{Z})$ can be expressed as [36]:

$$
Z(f)=\sqrt{\frac{\left(1+S_{11}\right)^{2}+S_{21}^{2}}{\left(1-S_{11}\right)^{2}-S_{21}^{2}}}=\frac{1+S_{11}}{1-S_{11}}
$$

In order to make sure the input impedance matches the free space impedance at every absorption frequency, the parameters of unit cell are optimized as shown in Fig. 2. There exist multiple resonant absorption peaks in tri-layer absorber from Fig. 2(a), and the first four absorption peaks (A, B, C and D) due to the higher absorption are mainly focused on. With decreasing the thickness of the dielectric layer $t_{2}$, the absorption bandwidth is increasingly narrow and each of the absorption peaks has a blue shift. When $t_{2}=0$, the structure of the absorber is simplified in Fig. 1(b), and peaks A, B, C and D are close to perfect absorbing. Next, the dependences of the radius $r$ and the thickness $t_{3}$ of the silicon disk on the absorption spectra are shown in Fig. 2(b) and Fig. 2(c), respectively. When the radius $r$ and the thickness $t_{3}$ gradually reduce, each of the resonant peaks also shows a blue shift. In these two figures, the absorptions of peaks $\mathrm{A}$ and $\mathrm{D}$ remain nearly constant. While the absorption of peak B is increasing followed with a decrease in $r$ and $t_{3}$, the maximum absorption of peak $\mathrm{C}$ appears at $t_{3}=3.6 \mu \mathrm{m}$. Based on the above analyses, the optimized values are chosen as follows: $t_{2}=0, t_{3}=3.6 \mu \mathrm{m}, r=35 \mu \mathrm{m}$.

Fig. 2(d) presents the absorption spectra for the TE and TM polarizations. It is observed that the two curves are absolutely identical due to the rotational symmetry of the structure. This illustrates that the absorber has a polarization insensitive. Four absorption peaks are located at center frequencies of $4.682 \mathrm{THz}, 5.150 \mathrm{THz}, 5.310 \mathrm{THz}$, $5.814 \mathrm{THz}$ with absorptions $99.11 \%, 99.85 \%, 99.70 \%, 99.93 \%$ respectively. Their full width at half maximum (FWHM) are $0.049 \mathrm{THz}, 0.039 \mathrm{THz}, 0.053 \mathrm{THz}, 0.078 \mathrm{THz}$, respectively. Fig. 2(e) shows the normalized input impedance $(Z)$ of the absorber calculated by formula (1). The $\mathrm{Z}$ at four absorption peaks $\mathrm{A}, \mathrm{B}, \mathrm{C}$ and $\mathrm{D}$ read $0.7990+i 0.0408,1.0758-i 0.0565,0.9437-i 0.1139$ and $0.9215+i 0.0740$, respectively. The real part of the $\mathrm{Z}$ is approximately equal to unity, and the imaginary part tends to zero. Therefore, the impedance of the device is fairly matched with the free-space 
impedance at four absorption peaks.

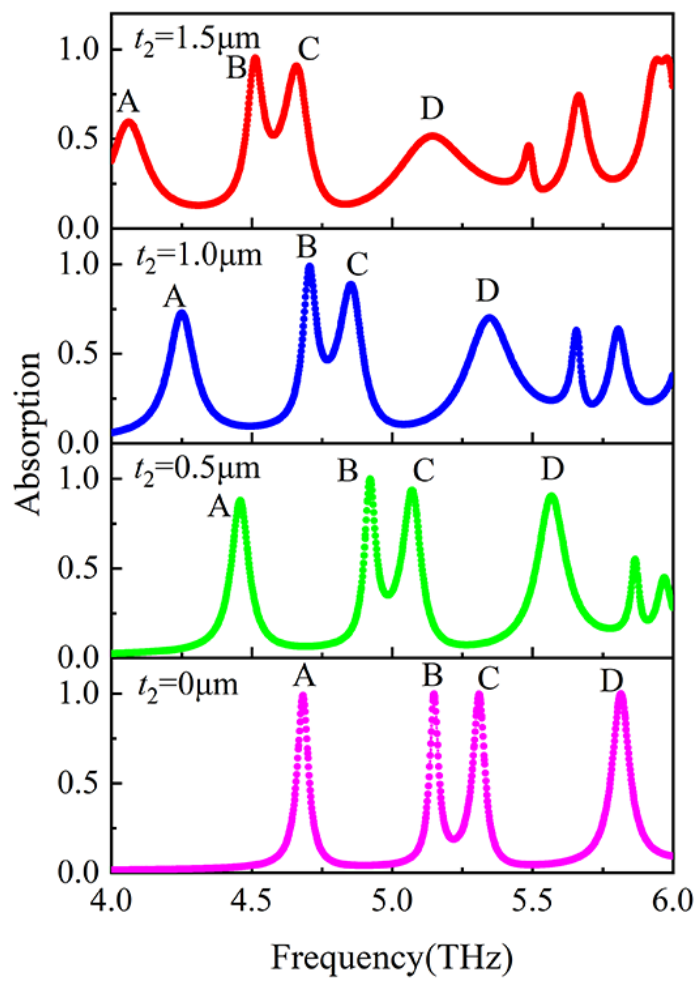

(a)

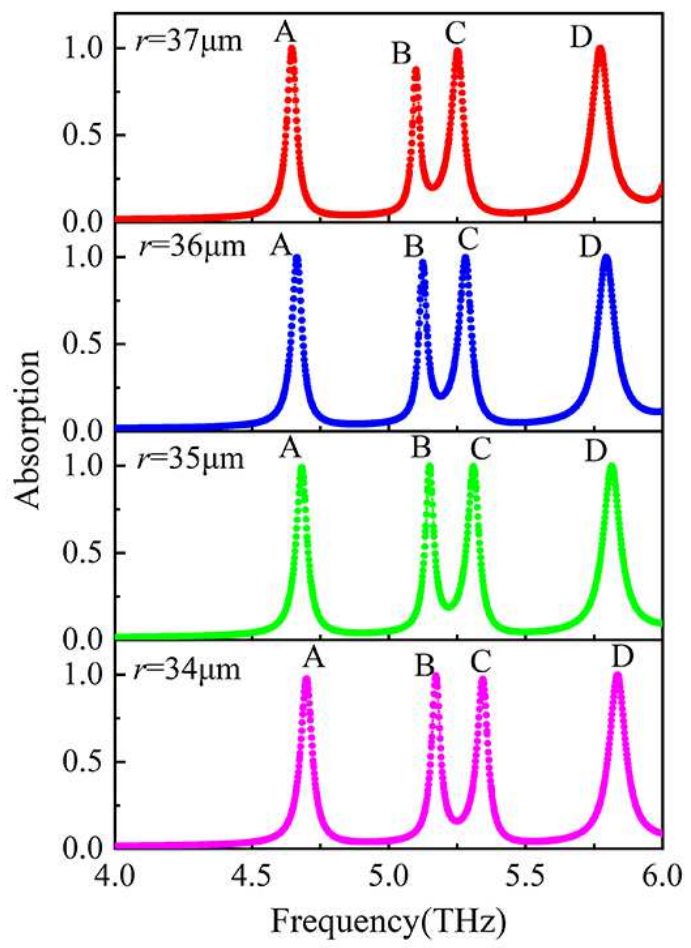

(c)

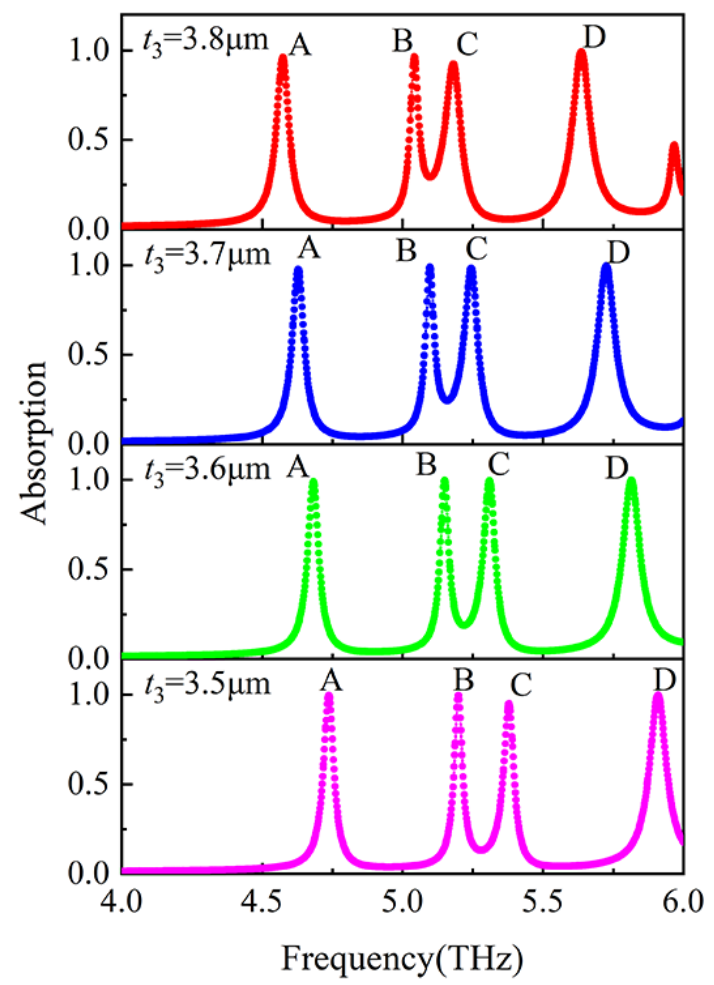

(b)
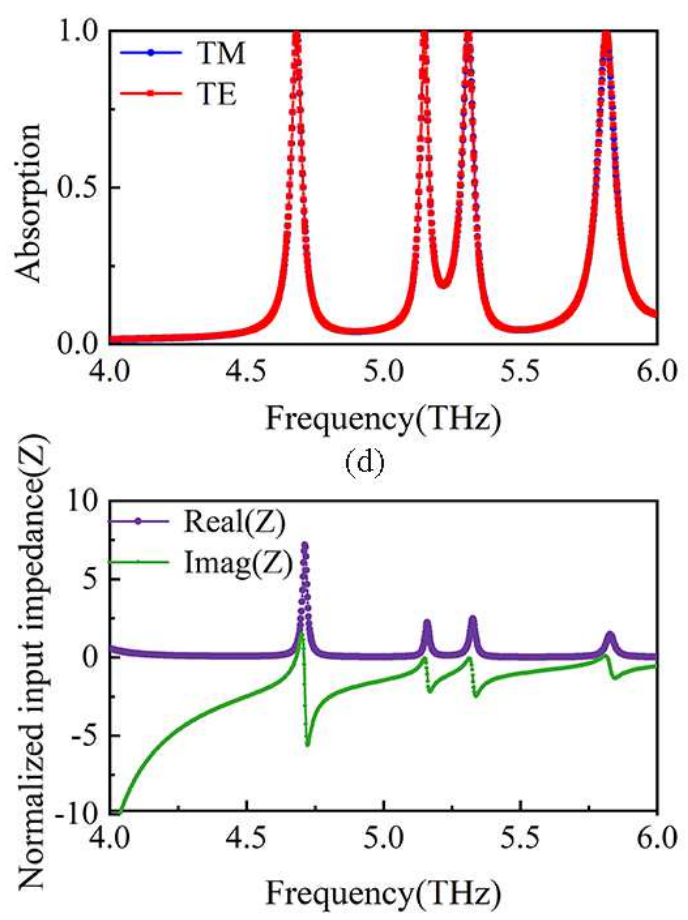

(e) 
Fig. 2 Absorption spectra with different thicknesses of the dielectric layer $t_{2}(\mathrm{a})$, the thickness $t_{3}$ (b) and the radius $r$ (c) of the silicon disk. (d) Absorption spectra for the TE and TM polarizations. (e) The normalized input impedance of the absorber.

To understand the physical mechanism of the absorber, the electric and magnetic field distributions at the frequencies corresponding to four absorption peaks are calculated, respectively. Fig. 3(a)-(d) represent the electric field distributions in the plane of $z=3.8 \mu \mathrm{m}$. And Fig. 3(e)-(h) illustrate the magnetic field distributions in the plane of $x=0 \mu \mathrm{m}$. It can be concluded from Fig. 3(a) that the accumulation of the charges in the silicon disk center leads to the excitation of the fundamental dipole. The opposite currents located between the silicon disk and gold layer excite the magnetic dipole [37] as shown in Fig. 3(e). The simultaneous excitation of the electric and magnetic dipole resonances of the silicon disk forms the fundamental absorption peak A. The appearance of higher order modes is due to the fact that the disk diameter is larger than a multiple of the half-wavelength of the resonant mode. The electric field distribution in Fig. 3(b) shows the excitation of multiple half wavelength charge oscillations in the silicon disk. This is the first higher order mode corresponding to the absorption peak B. Meanwhile, the charge distribution causes a multiple current circuits between the disk and gold layer, which gives rise to the excitation of multiple magnetic dipoles in the disk. The magnetic field is localized in the silicon disk [38] as shown in Fig. 3(f). The higher order electromagnetic resonance will result in absorption peaks in higher frequencies. Therefore, the structure has a absorption peak $\mathrm{C}$ at 5.31THz because of the second higher order mode and the corresponding electric and magnetic field distributions are shown in Fig. 3(c) and Fig. 3(g), respectively. Similarly, Fig. 3(d) corresponds to the third higher order mode with a absorption peak $\mathrm{D}$, and the silicon gives rise to excitation of three magnetic dipoles as shown in Fig. $3(h)$. 


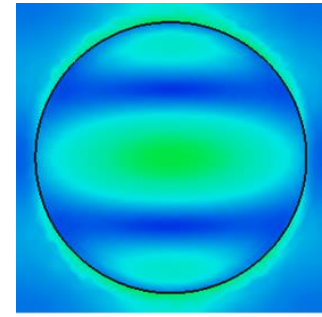

(a)

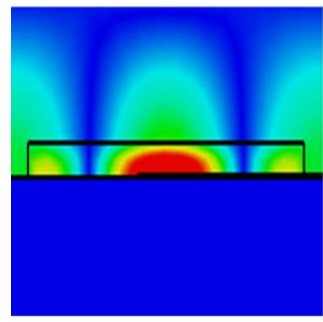

(e)

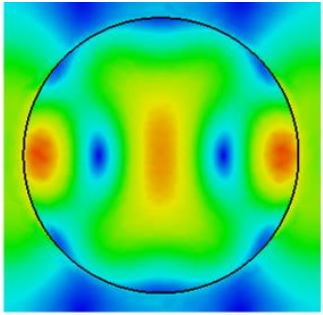

(b)

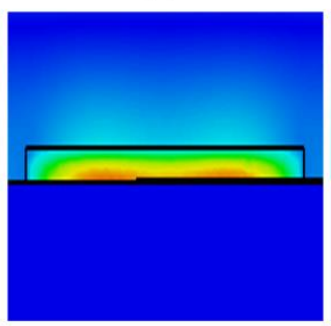

(f)

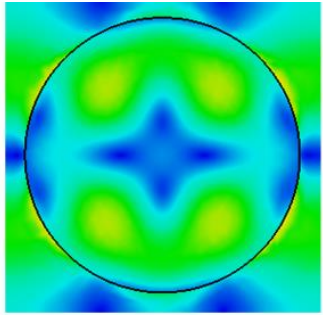

(c)

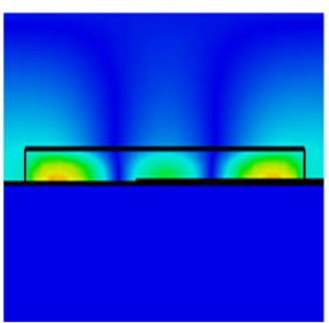

(g)

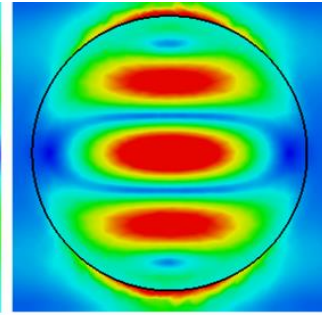

(d)

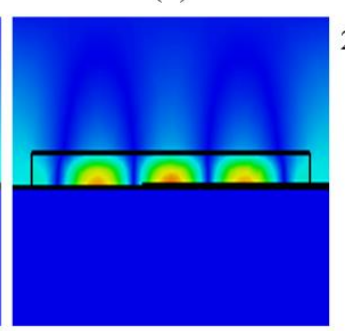

(h)
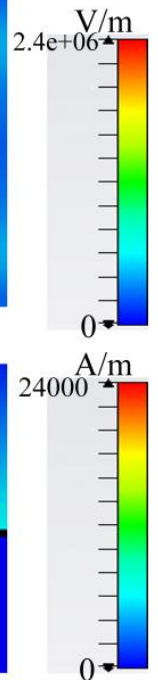

Fig. 3 The electric and magnetic field distributions of the absorber at the resonant frequencies of $4.682 \mathrm{THz}, 5.150 \mathrm{THz}, 5.310 \mathrm{THz}, 5.814 \mathrm{THz}$, respectively. The electric field distribution (a)-(d) in the plane of $z=3.8 \mu \mathrm{m}$, and the magnetic field distribution (e)-(h) in the plane of $x=0 \mu \mathrm{m}$.

\section{Analysis of the sensing performance}

The quality factor $\mathrm{Q}$ (which is defined as $Q=f_{0} / F W H W$, where $f_{0}$ is the center frequency of the absorption peak) is an important factor to estimate resonant modes, directly reflecting whether a resonant mode is applicable to sensing filed [30]. The higher the $\mathrm{Q}$ value, the better the sensing performance. The $\mathrm{Q}$ values of the resonant peaks A, B, C and D are 95.55, 132.05, 100.19, 74.54, respectively. Thus, the device is suitable for terahertz sensor. In general, the sensitivity $S$ and the FOM (figure of merit) are used to estimate the sensor quality and defined as [22, 39]:

$$
\begin{gathered}
S=\frac{\Delta f}{\Delta n} \\
F O M=\frac{S}{F W H M}
\end{gathered}
$$

where $\Delta f$ is a frequency shift as the refraction index $\Delta n$ unit changes.

First, the influences of refractive index (RI) of the analyte on four absorption peaks are analyzed. With the RI changing from 1.00 to 1.10 and a fixed thickness 
$(d=1 \mu \mathrm{m})$, the frequency shifts (FS) of four peaks are calculated in Fig. 4(a). Data fitting discovers a linear relationship between the RI of the analyte and the FS. The slope of the linear fitting equation is the sensitivity $S$ [40]. The sensitivities of peaks A, $\mathrm{B}, \mathrm{C}$ and $\mathrm{D}$ are $S_{A}=0.323 \mathrm{THz} / \mathrm{RIU}, \quad S_{B}=0.178 \mathrm{THz} / \mathrm{RIU}, \quad S_{C}=0.471 \mathrm{THz} / \mathrm{RIU}$, $S_{D}=0.462 \mathrm{THz} / \mathrm{RIU}$, respectively. The FOMs are $6.532 \mathrm{RIU}^{-1}, 4.564 \mathrm{RIU}^{-1}, 8.887 \mathrm{RIU}^{-1}$, $5.923 \mathrm{RIU}^{-1}$, respectively. Therefore, with the highest values of $S$ and $F O M$, peak $\mathrm{C}$ is most applicable for the RI sensor. Fig. 4(b) shows the absorption spectrum shifts of peak $\mathrm{C}$ under different RI. It is observed that the increase of the RI can result in strongly red shift of the absorption spectra.

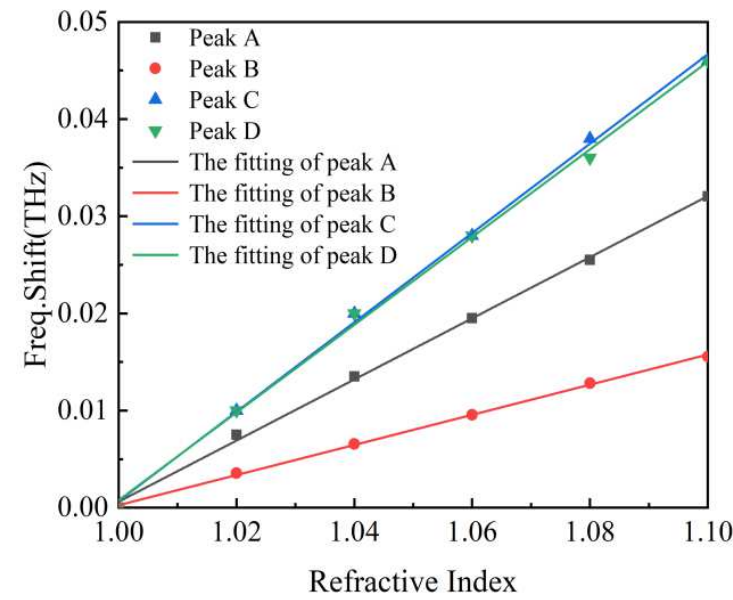

(a)

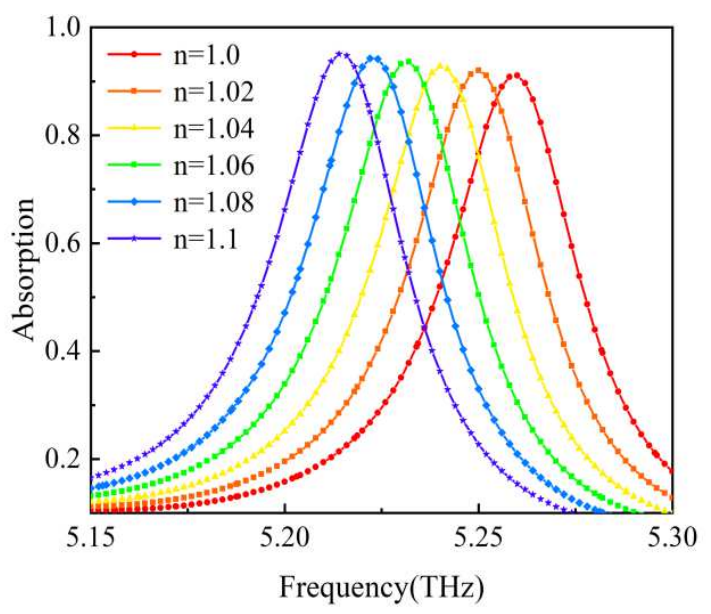

(b)

Fig. 4 (a) The FSs of four peaks with the RI of analyte varying from 1.00 to 1.10. (b) The absorption spectrum of peak $\mathrm{C}$ with different refractive index.

Next, the influences of the analyte thickness on four absorption peaks are investigated in detail. The RI of analyte keeps $n=1.04$. It is shown from Fig. 5(a) that with the increase of the thickness $d$, peak $\mathrm{C}$ will gradually disappear and the red shift of peak D is far smaller than those of peaks A and B. Therefore, peaks C and D are not suitable for the thickness sensor. Furthermore, the FSs of peaks A and B are calculated in Fig. 5(b), with the fitting functions $F S_{A}=1.201-1.798 \times \exp (-d / 11.896)$ and $F S_{B}=1.464-1.466 \times \exp (-d / 15.346)$, respectively. Apparently, the relationship between the anaylty thickness and the FS filed the exponential function. Also, the calculated results in Fig. 5(c) reveal that the sensitivities of peaks $A$ and $B$ filed the exponential functions, with the forms of 
$S_{A}=30.021-29.961 \times \exp (-d / 11.896)$ and $S_{B}=36.594-36.655 \times \exp (-d / 15.346)$, respectively. The highest sensitivities of peaks A and B are 30.021THz/RIU and 36.594THz/RIU with the FOMs $612.673 \mathrm{RIU}^{-1}$ and $938.308 \mathrm{RIU}^{-1}$, respectively. By combining the above analysis results, peak B is ideal for the thickness sensor.

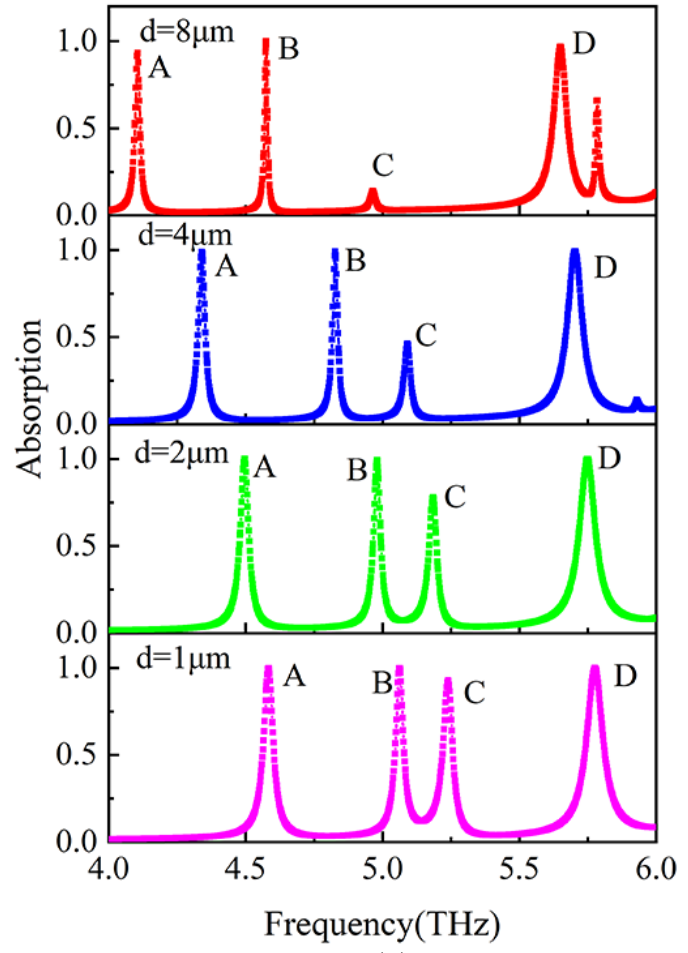

(a)

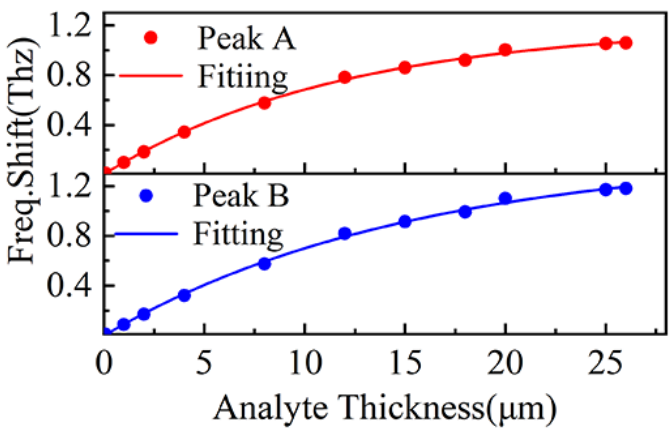

(b)

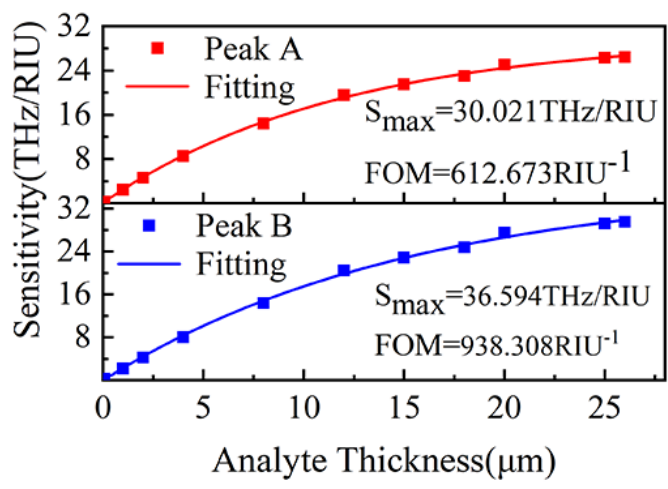

(c)

Fig. 5 Under different analyte thickness, absorption spectra of proposed absorber (a), the frequency shifts of peaks A and B (b) and the sesitivities of peaks A and B (c).

In conclusion, a novel multiband bi-layer ultra-thin terahertz metamaterial absorber with total thickness of $3.8 \mu \mathrm{m}$ is proposed. Simulation results show that the absorber has four resonant peaks with the absorption of each resonant peak over $99 \%$ in the 4.5 THz-6.0 THz frequency range. Due to the higher $\mathrm{Q}$ of four resonant peaks, the application of the proposed absorber in sensing fields is explored. It is found that the refractive index and thickness sensitivities of the device are $0.471 \mathrm{THz} / \mathrm{RIU}$, 36.594THz/RIU and the FOMs are $8.887 \mathrm{RIU}^{-1}, 938.308 \mathrm{RIU}^{-1}$, respectively. The proposed absorber has multiband prefect absorption and higher sensitivities of the refractive index and thickness, making great applications in multi-spectral imaging, photodetector and biosensors. 


\section{Acknowledgments}

This work was supported by the Education Commission Research Project of Tianjin (Grant No. 2018KJ213).

\section{Declarations}

\section{Funding}

The authors did not receive support from any organization for the submitted work.

\section{Conflicts of interest}

The authors have no financial or proprietary interests in any material discussed in this article.

\section{Availability of data and materials}

The datasets generated during and/or analysed during the current study are available from the corresponding author on reasonable request.

\section{Code availability}

Not applicable.

\section{Authors Contributions}

All authors contributed to the study conception and design. Material preparation, data collection and analysis were performed by Jinjun Bai, Wei Shen, Shasha Wang, Meilan Ge, Tingting Chen, Pengyan Shen and Shengjiang Chang. The first draft of the manuscript was written by Wei Shen and all authors commented on previous versions of the manuscript. All authors read and approved the final manuscript.

\section{Ethical Approval}

Not applicable.

\section{Consent to Participate}

Not applicable.

\section{Consent to Publish}

Not applicable. 


\section{References}

1. P. C. Theofanopoulos, M. Sakr, and G. C. Trichopoulos, "Multistatic Terahertz Imaging Using the Radon Transform," IEEE Trans. Antennas Propag. 67(4), 2700-2709 (2019).

2. T. Nagatsuma, G. Ducournau, and C. C. Renaud, "Advances in terahertz communications accelerated by photonics," Nature Photonics 10(6), 371-379 (2016).

3. X. Yang, X. Zhao, K. Yang, Y. P. Liu, Y. Liu, W. L. Fu, and Y. Luo, "Biomedical Applications of Terahertz Spectroscopy and Imaging," Trends in Biotechnology 34(10), 810-824 (2016).

4. C. M. Watts, X. Liu, and W. J. Padilla, "Metamaterial Electromagnetic Wave Absorbers," Advanced Materials 24(23), 98-120 (2012).

5. H. Tao, N. I. Landy, C. M. Bingham, X. Zhang, R. D. Averitt, and W. J. Padilla, "A metamaterial absorber for the terahertz regime: Design, fabrication and characterization," Optics Express 16(10), 7181-7188 (2008).

6. L. Shi, J. Shang, Z. Liu, Y. Li, G. Fu, X. Liu, P. Pan, H. Luo, and G. Liu, "Ultra-narrow multi-band polarization-insensitive plasmonic perfect absorber for sensing," Nanotechnology 31(46)(2020).

7. J. Wang, T. Lang, Z. Hong, T. Shen, and G. Wang, "Tunable terahertz metamaterial absorber based on electricity and light modulation modes," Optical Materials Express 10(9), 2262-2273 (2020).

8. L. J. Meng, D. Zhao, Z. C. Ruan, Q. Li, Y. Q. Yang, and M. Qiu, "Optimized grating as an ultra-narrow band absorber or plasmonic sensor," Opt. Lett. 39(5), 1137-1140 (2014).

9. Z. Liu, L. Guo, and Q. Zhang, "Design of Dual-Band Terahertz Perfect Metamaterial Absorber Based on Circuit Theory," Molecules (Basel, Switzerland) 25(18)(2020). 
10. G. S. Deng, T. Y. Xia, J. Yang, and Z. P. Yin, "Triple-band polarisation-independent metamaterial absorber at mm wave frequency band," Iet Microwaves Antennas \& Propagation 12(7), 1120-1125 (2018).

11. B. Zhang and J. Guo, "Optical properties of a two-dimensional nanodisk array with super-lattice defects," Journal of the Optical Society of America B-Optical Physics 30(11), 3011-3017 (2013).

12. Y. Ma, Q. Chen, J. Grant, S. C. Saha, A. Khalid, and D. R. S. Cumming, "A terahertz polarization insensitive dual band metamaterial absorber," Opt. Lett. 36(6), 945-947 (2011).

13. B. G. Xiao, M. Y. Gu, and S. S. Xiao, "Broadband, wide-angle and tunable terahertz absorber based on cross-shaped graphene arrays," Appl. Opt. 56(19), 5458-5462 (2017).

14. E. S. Torabi, A. Fallahi, and A. Yahaghi, "Evolutionary Optimization of Graphene-Metal Metasurfaces for Tunable Broadband Terahertz Absorption," IEEE Trans. Antennas Propag. 65(3), 1464-1467 (2017).

15. J. J. Bai, S. S. Zhang, F. Fan, S. S. Wang, X. D. Sun, Y. P. Miao, and S. J. Chang, "Tunable broadband $\mathrm{THz}$ absorber using vanadium dioxide metamaterials," Opt. Commun. 452(292-295 (2019).

16. J. F. Zhu, Z. F. Ma, W. J. Sun, F. Ding, Q. He, L. Zhou, and Y. G. Ma, "Ultra-broadband terahertz metamaterial absorber," Appl. Phys. Lett. 105(2), 4 (2014).

17. L. Liu, W. Liu, and Z. Song, "Ultra-broadband terahertz absorber based on a multilayer graphene metamaterial," J. Appl. Phys. 128(9)(2020).

18. A. Fardoost, F. G. Vanani, A. Amirhosseini, and R. Safian, "Design of a Multilayer Graphene-Based Ultrawideband Terahertz Absorber," IEEE Transactions on Nanotechnology 16(1), 68-74 (2017). 
19. J. Li, X. Chen, Z. Yi, H. Yang, Y. Tang, Y. Yi, W. Yao, J. Wang, and Y. Yi, "Broadband solar energy absorber based on monolayer molybdenum disulfide using tungsten elliptical arrays," Materials Today Energy 16((2020).

20. Y.-L. Liao and Y. Zhao, "A wide-angle broadband polarization-dependent absorber with stacked metal-dielectric grating," Opt. Commun. 370(245-249 (2016).

21. M. Mittendorff, S. Winnerl, J. Kamann, J. Eroms, D. Weiss, H. Schneider, and M. Helm, "Ultrafast graphene-based broadband THz detector," Appl. Phys. Lett. 103(2)(2013).

22. N. Liu, M. Mesch, T. Weiss, M. Hentschel, and H. Giessen, "Infrared Perfect Absorber and Its Application As Plasmonic Sensor," Nano Letters 10(7), 2342-2348 (2010).

23. M. Diem, T. Koschny, and C. M. Soukoulis, "Wide-angle perfect absorber/thermal emitter in the terahertz regime," Physical Review B 79(3)(2009)

24. Z. Zhou, T. Zhou, S. Zhang, Z. Shi, Y. Chen, W. Wan, X. Li, X. Chen, S. N. G. Corder, Z. Fu, L. Chen, Y. Mao, J. Cao, F. G. Omenetto, M. Liu, H. Li, and T. H. Tao, "Multicolor T-Ray Imaging Using Multispectral Metamaterials," Advanced Science 5(7)(2018).

25. J. Grant, I. J. H. McCrindle, C. Li, and D. R. S. Cumming, "Multispectral metamaterial absorber," Opt. Lett. 39(5), 1227-1230 (2014).

26. B.-X. Wang, X. Zhai, G.-Z. Wang, W.-Q. Huang, and L.-L. Wang, "A novel dual-band terahertz metamaterial absorber for a sensor application," J. Appl. Phys. 117(1)(2015).

27. Q. Xie, G. X. Dong, B. X. Wang, and W. Q. Huang, "Design of Quad-Band Terahertz Metamaterial Absorber Using a Perforated Rectangular Resonator for Sensing Applications," Nanoscale Research Letters 13(8(2018). 
28. R. Yahiaoui, S. Y. Tan, L. Q. Cong, R. Singh, F. P. Yan, and W. L. Zhang, "Multispectral terahertz sensing with highly flexible ultrathin metamaterial absorber," J. Appl. Phys. 118(8), 6 (2015).

29. M. G. Nielsen, A. Pors, O. Albrektsen, and S. I. Bozhevolnyi, "Efficient absorption of visible radiation by gap plasmon resonators," Optics Express 20(12), 13311-13319 (2012).

30. B. X. Wang, X. Zhai, G. Z. Wang, W. Q. Huang, and L. L. Wang, "Design of a Four-Band and Polarization-Insensitive Terahertz Metamaterial Absorber," Ieee Photonics Journal 7(1), 8 (2015).

31. X. P. Shen, T. J. Cui, J. M. Zhao, H. F. Ma, W. X. Jiang, and H. Li, "Polarization-independent wide-angle triple-band metamaterial absorber," Optics Express 19(10), 9401-9407 (2011).

32. G. Dayal and S. A. Ramakrishna, "Design of multi-band metamaterial perfect absorbers with stacked metal-dielectric disks," Journal of Optics 15(5)(2013).

33. S. Bhattacharyya, S. Ghosh, D. Chaurasiya, and K. V. Srivastava, "Bandwidth-enhanced dual-band dual-layer polarization-independent ultra-thin metamaterial absorber," Applied Physics a-Materials Science \& Processing 118(1), 207-215 (2015).

34. S. Liu, J. Zhuge, S. Ma, H. Chen, D. Bao, Q. He, L. Zhou, and T. J. Cui, "A bi-layered quad-band metamaterial absorber at terahertz frequencies," J. Appl. Phys. 118(24)(2015).

35. Y. Wang, M. Song, M. Pu, Y. Gu, C. Hu, Z. Zhao, C. Wang, H. Yu, and X. Luo, "Staked Graphene for Tunable Terahertz Absorber with Customized Bandwidth," Plasmonics 11(5), 1201-1206 (2016).

36. D. R. Smith, D. C. Vier, T. Koschny, and C. M. Soukoulis, "Electromagnetic parameter retrieval from inhomogeneous metamaterials," Physical Review E 71(3)(2005). 
37. S. Guddala, R. Kumar, and S. A. Ramakrishna, "Thermally induced nonlinear optical absorption in metamaterial perfect absorbers," Appl. Phys. Lett. 106(11), 5 (2015).

38. G. Dayal and S. A. Ramakrishna, "Multipolar localized resonances for multi-band metamaterial perfect absorbers," Journal of Optics 16(9), 6 (2014).

39. X. Lu, R. Wan, and T. Zhang, "Metal-dielectric-metal based narrow band absorber for sensing applications," Optics Express 23(23), 29842-29847 (2015).

40. L. Q. Cong, S. Y. Tan, R. Yahiaoui, F. P. Yan, W. L. Zhang, and R. Singh, "Experimental demonstration of ultrasensitive sensing with terahertz metamaterial absorbers: A comparison with the metasurfaces," Appl. Phys. Lett. 106(3), 5 (2015). 


\section{Figures}

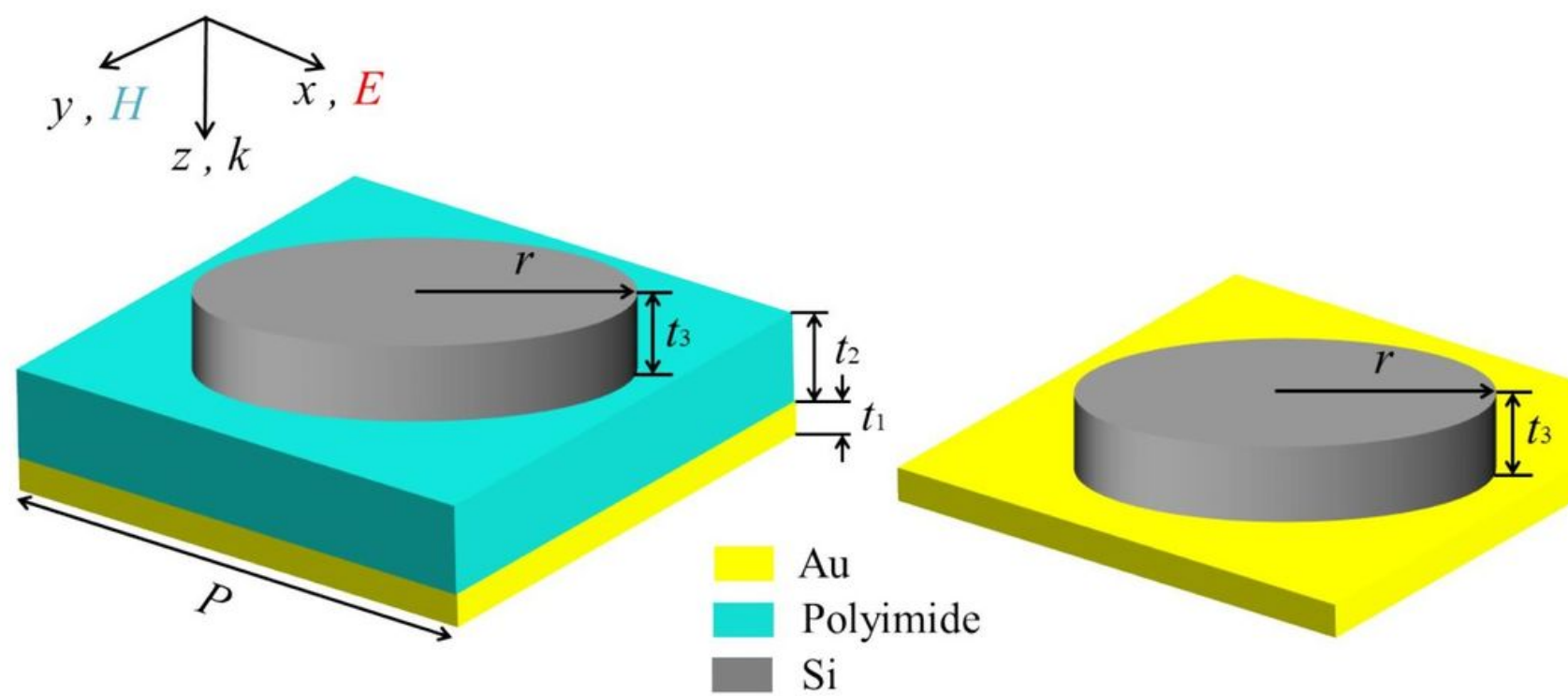

(a)

(b)

\section{Figure 1}

(a) The periodic structure schematic diagram of a tri-layer terahertz absorber. The top-down for a silicon disk (gray), polyimide (blue) and gold (yellow). (b) The periodic structure schematic diagram of a bi-layer terahertz absorber. 


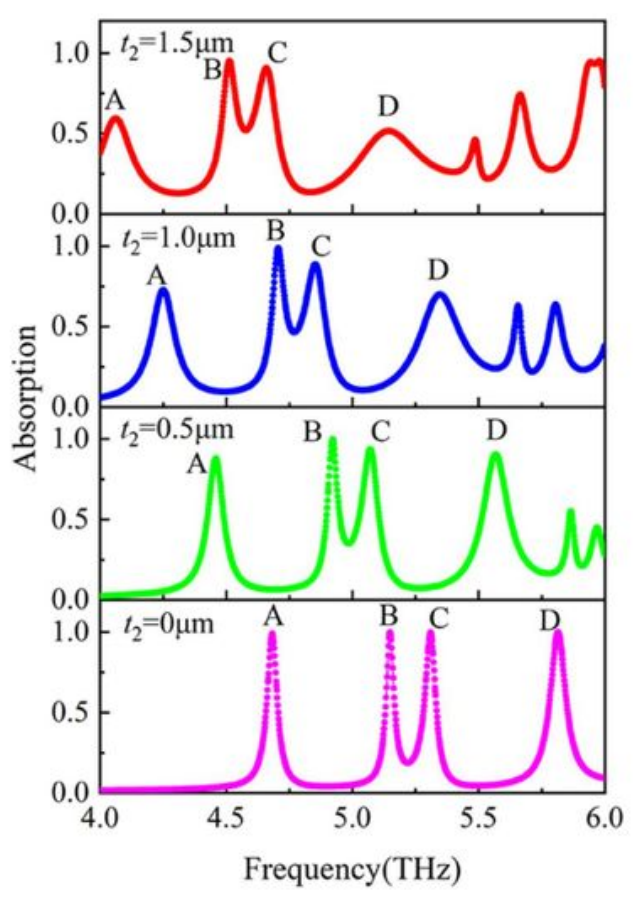

(a)

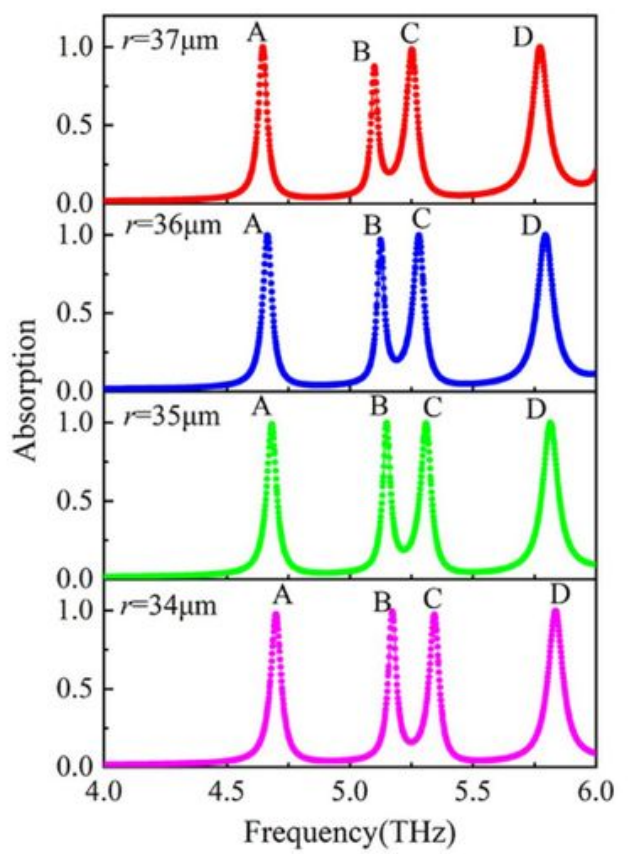

(c)

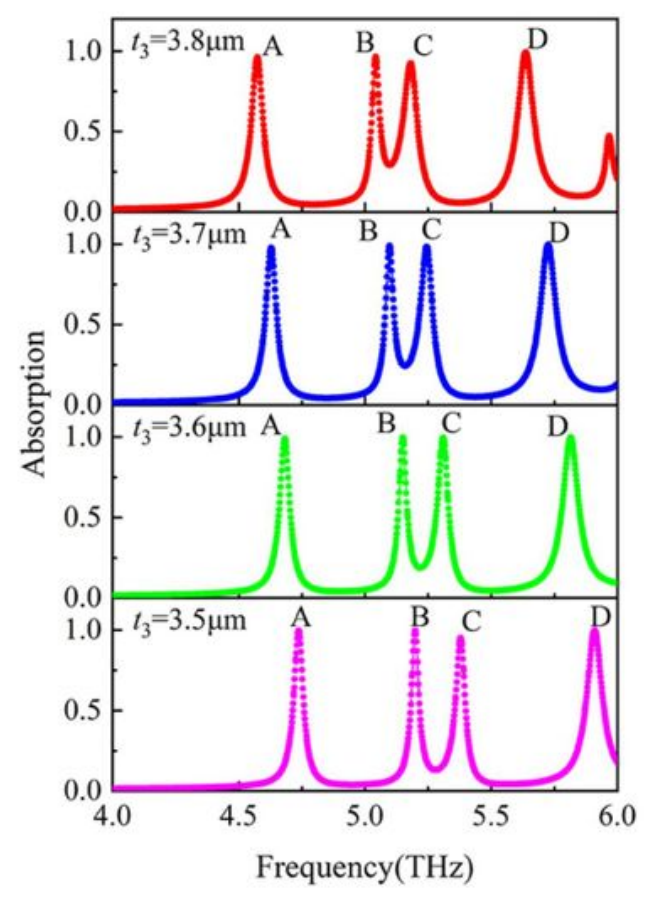

(b)

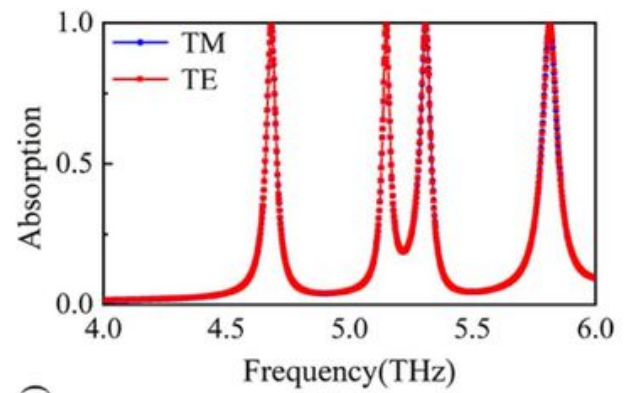

(d)

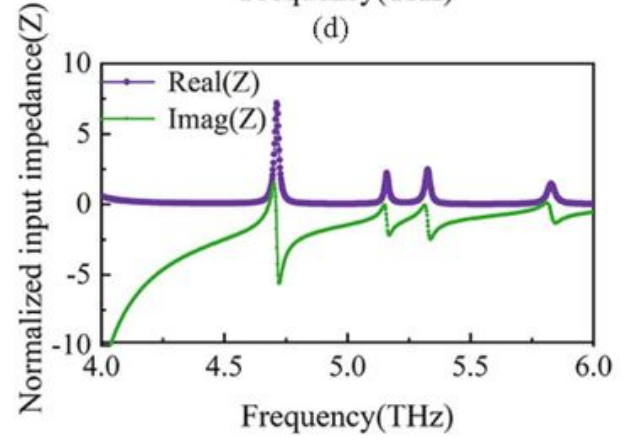

(e)

\section{Figure 2}

Absorption spectra with different thicknesses of the dielectric layer t2(a), the thickness $\mathrm{t} 3$ (b) and the radius $r$ (c) of the silicon disk. (d) Absorption spectra for the TE and TM polarizations. (e) The normalized input impedance of the absorber. 


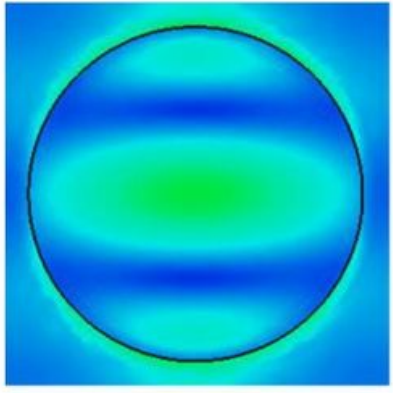

(a)

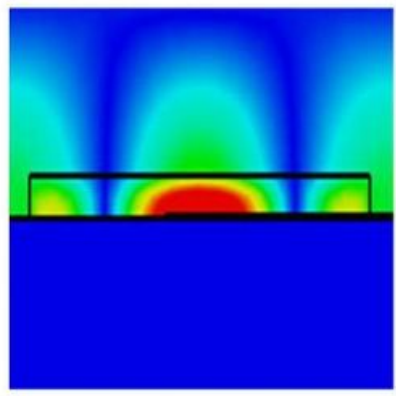

(e)

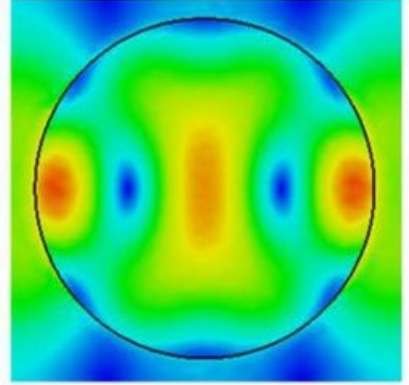

(b)

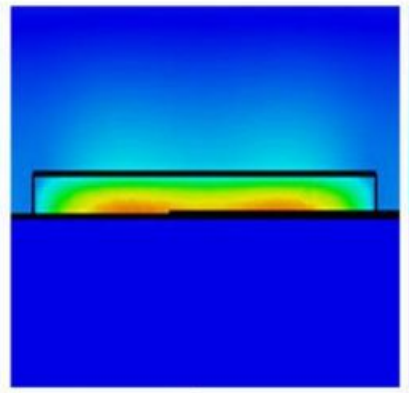

(f)

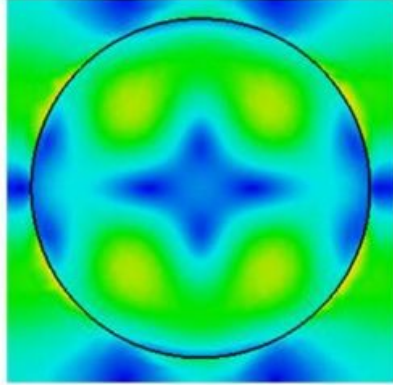

(c)

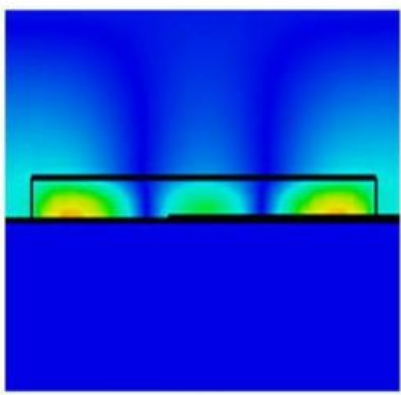

(g)

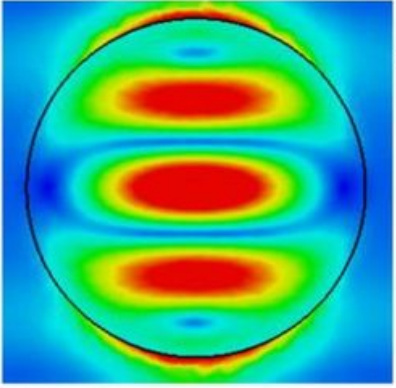

(d)

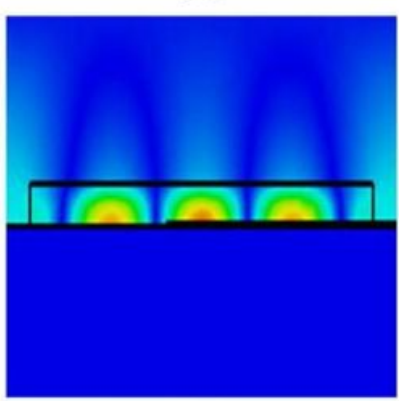

(h)
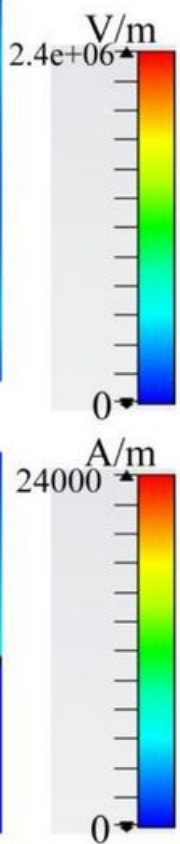

\section{Figure 3}

The electric and magnetic field distributions of the absorber at the resonant frequencies of 4.682THz, 5.150THz, 5.310 THz, 5.814THz, respectively. The electric field distribution (a)-(d) in the plane of z=3.8 $\mu \mathrm{m}$, and the magnetic field distribution (e)-(h) in the plane of $x=0 \mu m$

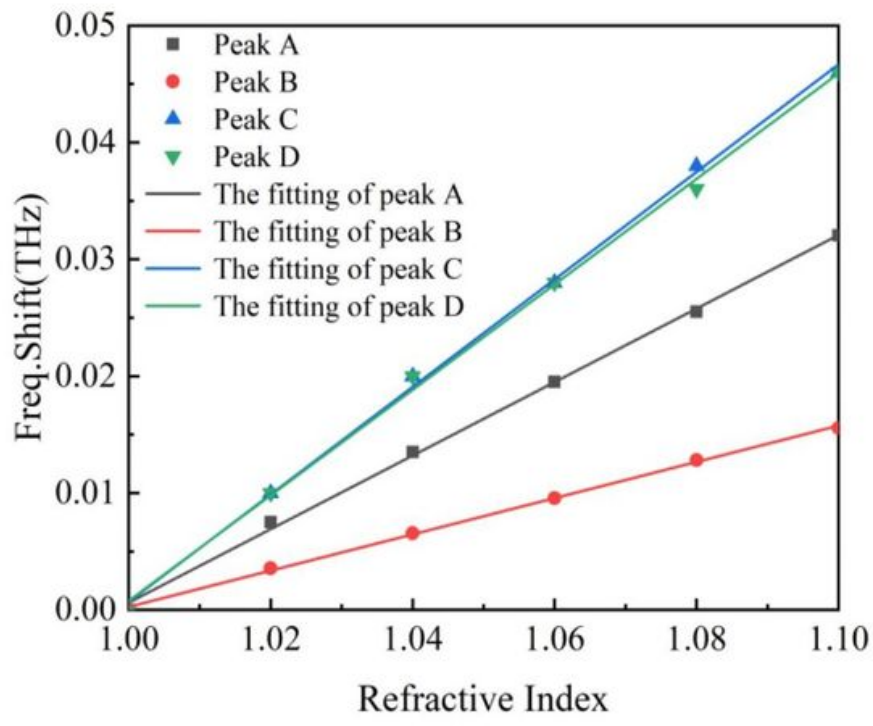

(a)

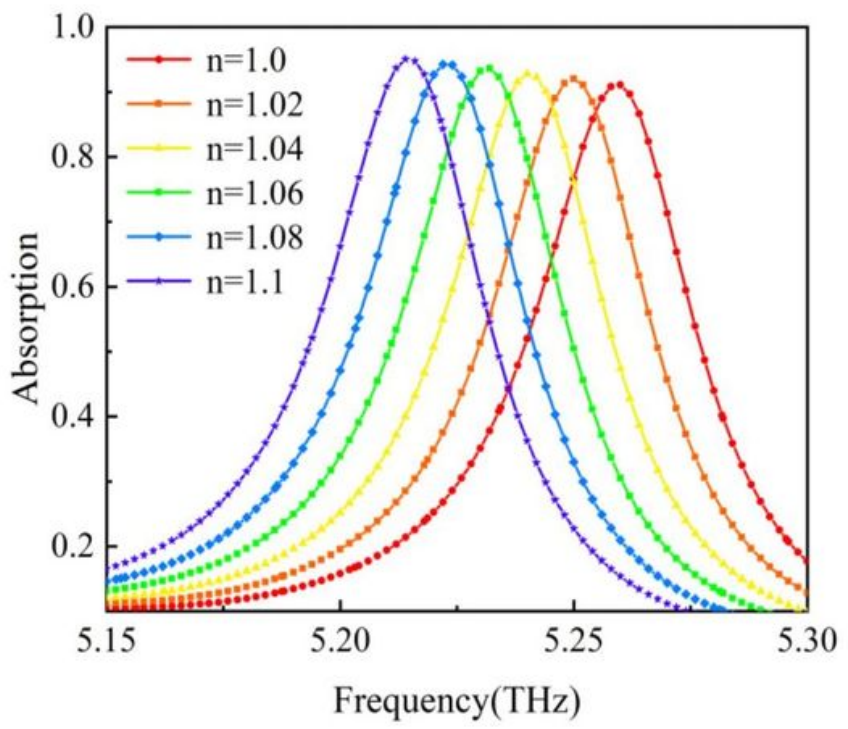

(b) 
(a) The FSs of four peaks with the RI of analyte varying from 1.00 to 1.10. (b) The absorption spectrum of peak $\mathrm{C}$ with different refractive index.

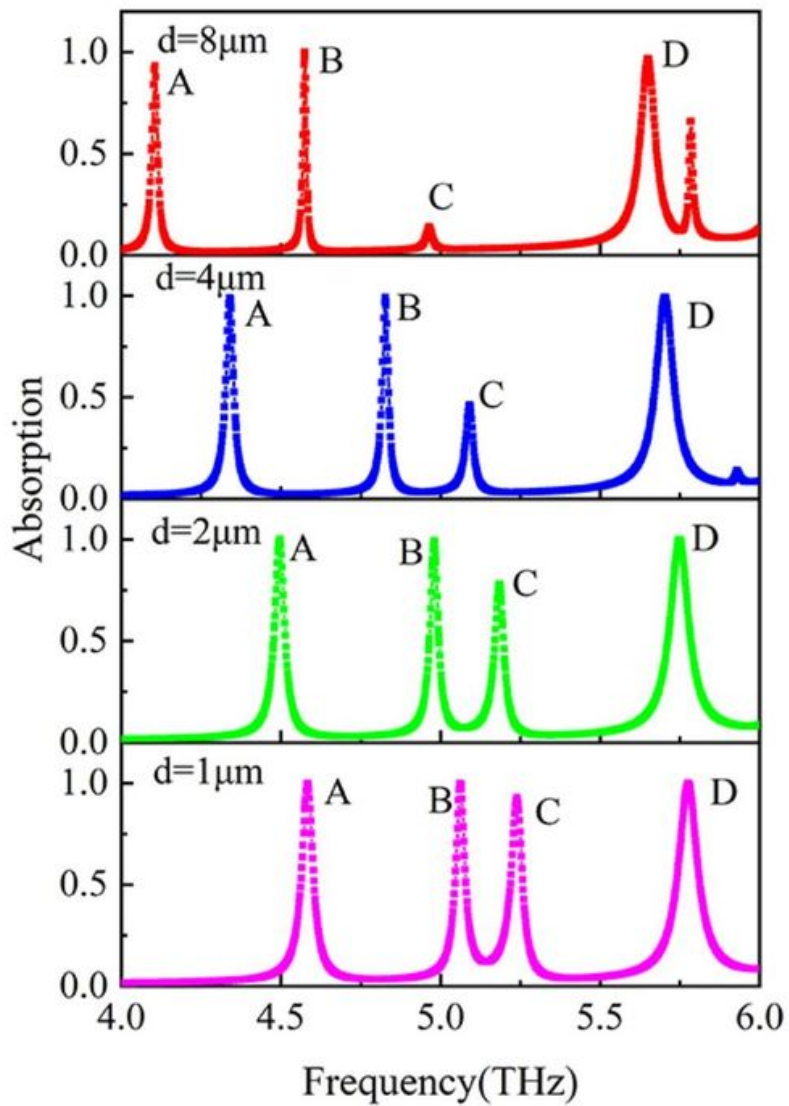

(a)

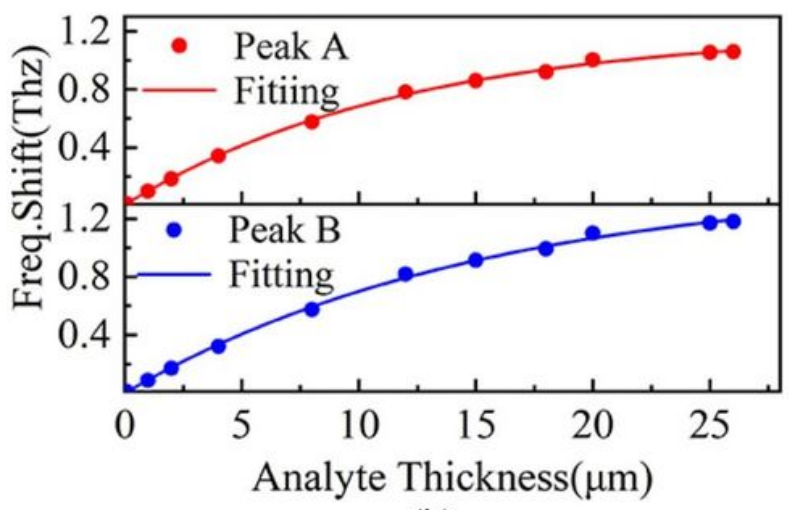

(b)

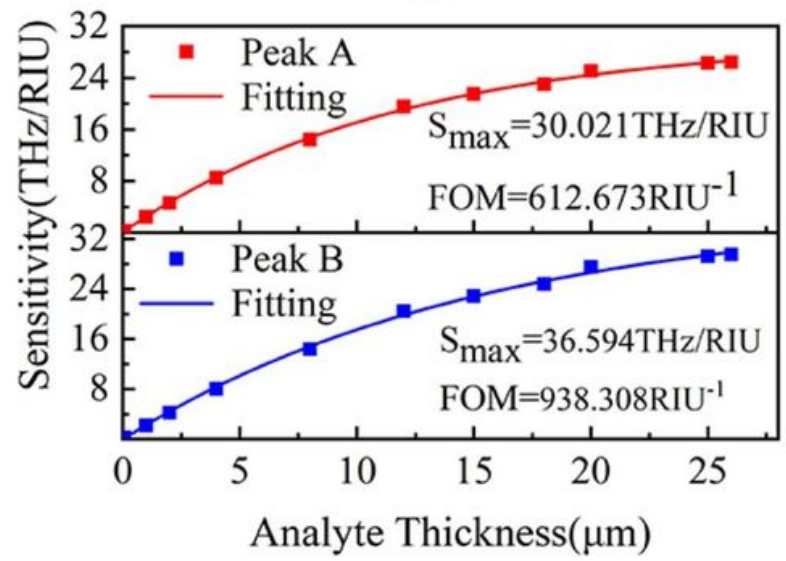

(c)

Figure 5

Under different analyte thickness $\$ absorption spectra of proposed absorber (a), the frequency shifts of peaks $A$ and $B(b)$ and the sesitivities of peaks $A$ and $B(c)$. 